\title{
Engineering Entrainment and Adaptation in Limit Cycle Systems
}

\author{
From biological inspiration to applications in robotics
}

Received: date / Revised version: date

\begin{abstract}
Periodic behavior is key to life, and is observed in multiple instances and at multiple time scales in our metabolism, our natural environment, and our engineered environment. A natural way of modelling or generating periodic behavior is done by using oscillators, i.e. dynamical systems that exhibit limit cycle behavior. While there is extensive literature on methods to analyze such dynamical systems, much less work has been done on methods to synthesize an oscillator to exhibit some specific desired characteristics. The goal of this article is two-fold: (1) to provide a framework for characterizing and designing oscillators, and (2) to review how classes of well known oscillators can be understood and related to this framework.
\end{abstract}

The basis of the framework is to characterize oscillators in terms of their fundamental temporal and spatial behavior, and in terms of properties that these two behaviors can be designed to exhibit. This focus on fundamental properties is important because it allows us to systematically compare a large variety of oscillators which might at first sight appear very different from each other. We identify several specifications that are useful for design, such as frequency-locking behavior, phaselocking behavior, and specific output signal shape. We also identify two classes of design methods by which these specifications can be met, namely off-line methods and on-line methods. By relating these specifications to our framework and by presenting several examples of how oscillators have been designed in the literature, this article provides a useful methodology and toolbox for designing oscillators for a wide range of purposes. In particular the focus on synthesis of limit cycle dynamical systems should be useful both for engineering and for computational modelling of physical or biological phenomena.

Biologically Inspired Robotics Group

School of Computer \& Communication Sciences

Ecole Polytechnique Fédérale de Lausanne

INN, Station 14, 1015 Lausanne, Switzerland

E-mail: jonas.buchli@epfl.ch

\section{Introduction}

Periodic behavior is central to our lives. Our body functions thanks to many types of periodic behaviors ranging from heart beats, breathing, chewing, locomotion, various rhythms in the brain, down to cycles in gene regulatory networks. Similarly our natural environments have multiple periodic phenomena such as rotations of the earth around the sun and around itself, seasons, tides, cycles in ecological systems (e.g. prey-predator populations), in chemical reactions, etc. Finally, many systems that we engineer are meant to exhibit periodic behavior such as clocks (for watches or CPUs), lasers, music, traffic lights, satellites, to name just a few examples. All these phenomena share many common features, and can be modelled (or controlled for engineered systems) by systems of differential equations that exhibit limit cycle behavior, that is by oscillators.

The importance and ubiquity of periodic behavior explains why oscillator models are published in such a large variety of journals in different fields (nonlinear dynamics, physics, biology, chemistry, engineering, etc.). This makes oscillators a very exciting topic of study, but at the same time makes it difficult to extract common principles from all these models. Indeed each field has its own terminology, variable/parameters names, systems of coordinates, methods of analysis, methods of synthesis and this makes it hard to see similarities and differences between models. Furthermore, the choice of a particular oscillator in a given field is often not transparent, and depends sometimes more on historical reasons than on pure design or modelling considerations. The reason for that is usually a lack of abstraction of the concept of oscillators. Finally, another difficulty with oscillators is that while there are many tools for analyzing the behavior of an oscillator (see (Kuramoto, 1984; Winfree, 2001; Glass \& Mackey, 1988; Pikovsky, Rosenblum, \& Kurths, 2001) for some outstanding textbooks), there is a lack of methodologies for designing them to exhibit a particular behavior. 
The goal of this article is therefore to provide a framework for characterizing different oscillator models in a systematic way, and to focus on methodologies that can be used for designing them. In this process, we review a large range of oscillator models that have been developed as well as some of our own work in adaptive frequency oscillators. We will try to focuss on the fundamental principles of limit cycle systems, and separate those from unnecessary details of a particular implementation. These principles can then be applied to the design of systems, and used to provide guidelines of how to endow a system with a set of predefined properties. Our perspective is therefore mainly an engineering one, i.e. we want to address the problem of how an oscillator or a system of coupled oscillators can be designed to do something useful (e.g. for coordination, sequencing, and/or pattern formation), but the approach should also be interesting for computational modelling.

Oscillators are of interest for engineers for several reasons. They can be exploited for timing and sequencing. They can synchronize to external signals, and show coordinated behavior with perturbations and other oscillators. Connecting them into networks or latices they can form coordinate yet flexible spatio-temporal patterns. These networks can act as pattern generators which can reduce the dimensionality of a given control problem, in the sense that a small number of simple (scalar) parameters can control multidimensional output patterns. Of course they also exhibit all the common features of structurally stable dynamical systems such as smooth changes under parameter variation. The structurally stability makes it possible to fuse in input without destroying the autonomous dynamics of the system, i.e. the resulting dynamics is a combination of internal and external dynamics.

An interesting example of the use of oscillators in engineering is in the field of locomotion control in robots. Locomotion control is still a difficult and unsolved problem for robots with multiple degrees of freedom (e.g. legged robots). Locomotion requires multi-dimensional coordinated periodic patterns that need to to satisfy multiple constraints in terms of efficient locomotion, energy and adaptation to complex terrain. One approach to solve this problem relies on accurate models of the robot and environment dynamics to develop control laws for locomotion. These model-based methods have however significant difficulties dealing with environments that are hard to model properly (e.g. with complex terrains). An alternative approach is to use systems of coupled oscillators and to take inspiration from animal motor control. In vertebrate animals, an essential building block of the locomotion controller is the central pattern generator (CPG) located in the spinal cord. A CPG is a neural circuit capable of producing coordinated patterns of rhythmic activity in open loop, i.e. without any rhythmic inputs from sensory feedback or from higher control centers (Delcomyn, 1980; Grillner, 1985). A CPG can be modelled as a system of coupled oscillators (Kopell \& Ermentrout, 1988; Kopell, 1988). The motivation for using CPG models in robotics is to produce the periodic patterns necessary for locomotion as limit cycles. If this is the case, the oscillatory patterns are robust against transient perturbations (i.e. they asymptotically return to the limit cycle), and this makes them well suited to deal with unexpected perturbations from the environment. Furthermore, the limit cycle can usually be modulated by some parameters, which offers the possibility to smoothly modulate the gait (e.g. increase frequency and/or amplitude) or even to induce gait transitions (i.e. bifurcations between different types of limit cycles, see for instance (Collins \& Richmond, 1994)). Finally, CPGs can readily integrate sensory feedback signals in the differential equations, and show interesting properties such as entrainment by the mechanical body (Taga, 1998). Because of these interesting properties, CPGs are increasingly used in robotics (see for instance (Kimura, Akiyama, \& Sakurama, 1999; Wilbur, Vorus, Cao, \& Currie, 2002; Endo, Nakanishi, Morimoto, \& Cheng, 2005; Buchli, Iida, \& Ijspeert, 2006; Righetti \& Ijspeert, 2006b)).

The paper is organized as follows. First, in Section 2 , we introduce oscillators (i.e. limit cycle systems) by definitions, and present a description of the very basic features common to all limit cycle systems. We then discuss the typical stability properties of oscillators, and this leads us to the formulation of two distinct coordinate systems, the Phase-Radius coordinate system and the Q coordinate system (the "physical" coordinate system), and their relationship. Examples of the abstract concepts by help of well known oscillators will be given along the way. This discussion helps us to get clear what properties of an oscillator can be designed. Based on this discussion we then address the issue how we can construct an oscillatory system to exhibit specific properties. This part addresses the three core questions: What can we do (Section 3)? How can we do it (Section 4)? What are the resulting systems (Section 5)? Finally, we conclude with a general discussion of design choices and give an outlook on future research in this direction.

We assume some familiarity with basic concepts of nonlinear dynamical systems, a good introduction can be found in e.g. in (Strogatz, 1994), especially the concept of stability will be used extensively. The mathematical facts presented in this article are often not new (many are on textbook level), however the way of presenting oscillators is new due to the focus of generic properties of all oscillators. This leads to a novel discussion of oscillators centered around the transformation between a canonical system in the Phase-Radius coordinate system in which it is particularly simple to discuss the influence of perturbation of oscillators, and the "physical" system, i.e. the traditional way of representing oscillators. To support the discussion the new concept of radius isochrones is introduced. Finally, we would like to give a note about mathematical detail and completeness. For 
the sake of clarity we do not discuss every subtlety and every case since we think that would dilute the clarity of the concepts. An excellent and very comprehensive introduction to oscillators and synchronization phenomena is (Pikovsky et al., 2001).

\section{Limit cycle systems}

In this section we will introduce the mathematical concept of an oscillator. As will get clear there are some subtle differences of what commonly is called an oscillator and the mathematical concept of an oscillator.

The presented concepts are key to all the design issues discussed later, such as the choice of type of oscillator, coupling etc. This means, an engineer wanting to use an oscillator needs a thorough understanding of those concepts. This will allow him/her to chose the right type of oscillator and gives him/her the tools to engineer its properties.

Definitions In order to support our mathematical discussion of oscillators we start with its definition.

Definition 1 An oscillator is a autonomous dynamical system, i.e. a system of differential equations with at least one limit cycle attractor. In other words the solution of the system (after a transient time) is a closed cycle, which is asymptotically stable, i.e. if the system gets perturbed out of the limit cycle it returns back to it.

We see that the limit cycle attractor is the defining property of an oscillator, hence the name limit cycle (LC) system is used as synonym for oscillator.

This means the system has a self-sustained oscillatory behavior to which it returns after a transient perturbation. Oscillators possess thus an intrinsic period (and hence frequency) with which the system repeats the pattern of activity. Thus, a linear 2nd (or higher) order system can not be an oscillator in that sense. It can only exhibit sustained oscillations with an oscillatory input in case the system is damped. If it is not damped, the system is just on the border between stability and instability and oscillations are not structurally stable (i.e. after a transient perturbation possibly another pattern is assumed). On the other side of the stability border the oscillations will increase to infinite amplitude.

Let us detail this definition a bit more, for this we need some nomenclature which we list in Table 1.

With the help of those variables we can put above definition in more concise terms:

Definition 2 (equivalent to Def. 1) If the dynamical system

$$
\dot{\mathbf{q}}=\mathbf{F}(\mathbf{q})
$$

has a stationary solution which is a closed curve and the solution is structurally stable, (1) is an oscillator.
$\mathbf{F}(\mathbf{q}, \rho)$ System of equations describing the dynamics of the system, $\mathbf{F}(\mathbf{q})=\left[f_{1}, \ldots, f_{D}\right]^{T}$

$\mathbf{q} \quad$ State vector $\mathbf{q}=\left[q_{1}, \ldots, q_{D}\right]^{T}$

$\rho \quad$ Vector of parameters

$\mathbf{p}(t) \quad$ Vector of perturbations

D Dimension of the system

$\phi \quad$ Phase of the oscillator

$\omega \quad$ Intrinsic frequency of the oscillator

$\mathbf{r} \quad$ Radius of the oscillator $\mathbf{r}=\left[r_{1}, \ldots, r_{D-1}\right]^{T}$

$T \quad$ Period of the oscillator

$\Omega_{i} \quad$ Instantaneous frequency

$\mathbf{e}_{\phi}, \mathbf{e}_{\mathbf{r}} \quad$ Unit vectors in direction of phase and radius

$\mathbb{T} \quad$ Transformation from $\mathbf{q}$ to $[\phi, \mathbf{r}]$

$\mathbf{q}_{\infty} \quad$ The set of points describing the limit cycle

PRCS Phase-radius coordinate system

QCS The q-coordinate system

LC Limit cycle

PS Phase sensitivity

PRC Phase response curve

Table 1 Nomenclature, conventions and common abvrevations used to discuss oscillators in this article.

The limit cycle The set on which $\mathbf{q}$ evolves is called the limit cycle, which we denote with $\mathbf{q}_{\infty}$. It is an attractor of dimension 1 (i.e. a curve) which is closed in itself, so it needs to be embedded in a space of dimension $D \geq 2$ (cf. Fig. 1). The fact that the attractor forms a closed curve implies that the time shift invariance holds: if $\mathbf{q}(t)$ is a solution then $q(t)=\mathbf{q}(t+n T), T$ is the time of repetition, i.e. the period of the system and is inversely proportional to the intrinsic frequency $\omega$ of the system $T=\frac{2 \pi}{\omega}$ (cf. Fig. 1b).

The fact of having a closed curve implies also a special stability property of the attractor. The flow described by the set of equations lets all solutions within the basin of attraction converge to the limit cycle. Perpendicular to the limit cycle the system is thus asymptotically stable. But the phase point moves along the limit cycle. In other words in every point on the limit cycle the flow is stable/contracting in to D-1 directions, but drives the state in the one direction perpendicular to the other directions. This is the very essence of a limit cycle system, these stability properties are the only ones that allow for a closed 1-dimensional attractor. And they are the key to understand the properties of oscillators their particular behavior and phenomena such as synchronization.

We can thus distinguish two characteristic stability directions on the limit cycle by introducing a coordinate system of which one basis vector is tangential to the limit cycle limit cycle, $\mathbf{e}_{\phi}$, and $D-1$ vectors perpendicular to the limit cycle, which we do denote representatively by $\mathbf{e}_{\mathbf{r}}$ (cf. Fig. 1a).

As we will see in the next section the marginally stable direction tangential to the limit cycle is of central importance to discuss oscillators.

The phase In every oscillator we can identify a variable (which does not necessarily correspond to a state variable but is a function of those) which grows uniformly in time 
a)

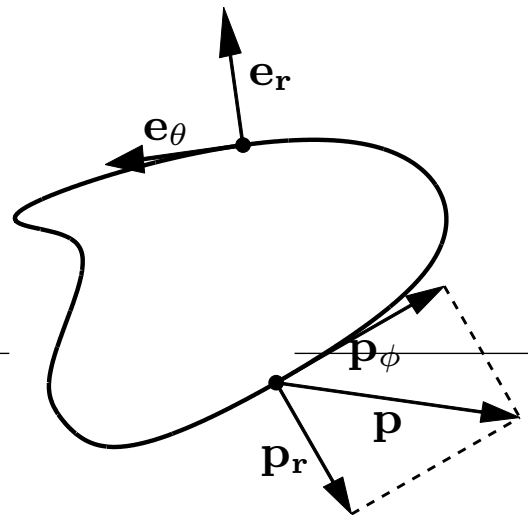

b)

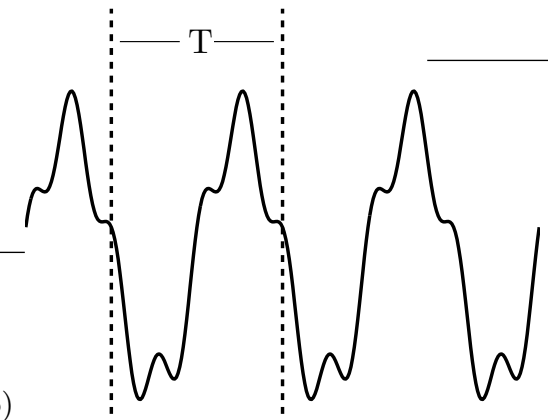

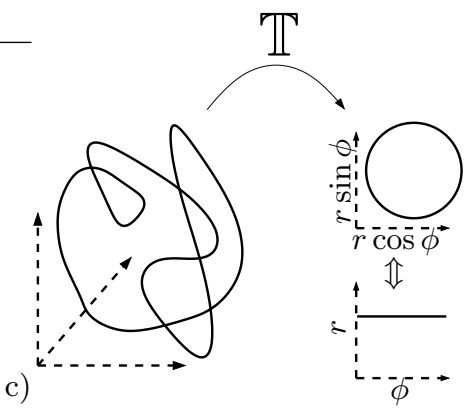

Fig. 1 a) The schematic illustration of a limit cycle. It is a closed curve in phase space. The stability directions $\mathbf{e}_{\phi}, \mathbf{e}_{\mathbf{r}}$ are illustrated as well as the projections $\mathbf{p}_{\mathbf{r}}$ and $\mathbf{p}_{\phi}$ of a perturbation $\mathbf{p}$, which has a direction in the phase space, onto those stability directions. b) The time series of an hypothetical oscillator. There is a characteristic period $T$ after which the activity of the oscillator and with this the time series repeat. c) The limit cycle is a 1-dimensional manifold embedded in a $D$-dimensional space $(D \geq 2)$, we can transform the system into a coordinate system in which the manifold shows particularly simple form and of which the stability directions constitute the base vectors.

and is interpreted $\bmod 2 \pi$ (or any other convention). This variable is called the phase of the oscillator.

The phase of the oscillator is a measure where the oscillator is in the cycle. We remember that the frequency of the oscillator is $\omega=\frac{2 \pi}{T}$. We define the phase in the following way

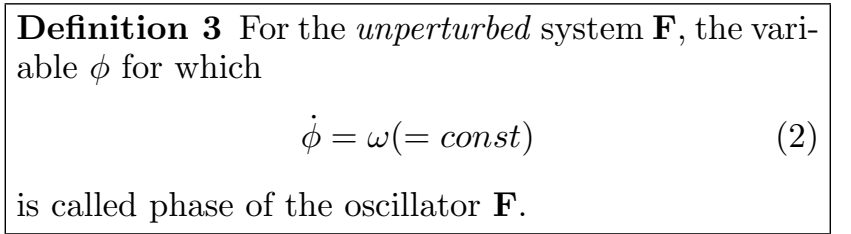

By help of the phase also the frequency can be cast into a definition

Definition 4 The rate of change $\omega$ of $\phi$ in the unperturbed oscillator is the intrinsic frequency of the oscillator.

The reason why we define the frequency by help of the phase will become clear later when we will discuss the oscillator under perturbations. It is important to note that $\omega$ is not always an explicit parameter. However, it is always a function of the parameters, $\omega=f(\rho)$.

Now, every oscillator can be transformed into a phase $(\phi)$-radius (r) coordinate system (Pikovsky et al., 2001):

$$
\begin{gathered}
\dot{\phi}=\omega \\
\dot{\mathbf{r}}=\mathbf{F}_{r}(\mathbf{r})
\end{gathered}
$$

where $\mathbf{F}_{r}$ is the dynamical system describing the evolution of $\mathbf{r}$ (refer to Fig. 1c) and has a stable fixed point.

The vectors $\mathbf{e}_{\phi}$ and $\mathbf{e}_{\mathbf{r}}$ that we introduced above based on stability considerations form the basis vectors of the phase radius coordinate system. In Eq. 3 the fact that the phase is a marginally stable variable $\left(\frac{d \dot{\phi}}{d \phi}=0\right)$ is immediately evident. This coordinate system is the natural one to discuss influences on the oscillator since the stability directions are decoupled.

At this stage it is also worth noting, that in a unperturbed oscillator the phase completely describes the state of the system in the stationary regime (cf. phase oscillators in Section 3.1). This means we can write $\mathbf{r}_{\infty}=f(\phi)$.

It is important to realize, that the phase is not necessarily proportional with time. This is only the case when the oscillator is unperturbed, where indeed $\phi$ is proportional to $t \bmod 2 \pi$ - but more importantly and this is the key to the entrainment effects as we will see later, in case of perturbations the phase and time "get decoupled" i.e. the phase can be shifted forward or backwards. The oscillations can be accelerated or de-accelerated. Designing entrainment and other aspects of the oscillator is all about designing these acceleration and de-acceleration effects. We will discuss this in more detail when we look at LCs under perturbations in Section 2.2. But first we need to complete our understanding of the phase-radius coordinate system.

The geometry of the limit cycle Normally the oscillator is not readily represented in the ideal phase-radius coordinate system (as in Eqs. 3-4). We need to discuss the relationship between the oscillators representation in $\mathbf{q}$ and in $[\phi, \mathbf{r}]$. As we will see that is the key to understand the behavior of the oscillator under perturbations.

If we transform the coordinate system and the metrics we could possibly gain a simpler oscillator but the complexity gets transfered into the coupling of the oscillator the input. We will give an example when we discuss phase oscillators in Section 3.1.

But then how is the coordinate system usually determined? Let us reflect on the role of the state variables. Usually the state variables are defined by a physical interpretation or they have a concrete conceptual meaning 
such as a voltage or a chemical concentration, for instance, and that is the way the coordinate system gets defined. This coordinate system is the natural one to formulate the physical laws and interaction between the different physical entities (while, as we have seen, phase radius is the natural one to discuss perturbation because of the separation of variables according to stability properties). And in modeling it is usually the case that the input and outputs of an oscillator are formulated in the physical coordinate system.

But, for engineering, if we want to exploit some of the abstract properties of the oscillator we are not bound to an interpretation of the variables. Thus it can be useful to formulate the inputs and outputs in the phase radius coordinate system or any other suitable coordinate system.

We name thus two coordinate systems, the phase radius coordinate system: PRCS and the coordinate system of q: QCS. The transformation from QCS to PRCS is given by $\mathbb{T}$.

$$
[\phi, \mathbf{r}]^{T}=\mathbb{T}(\mathbf{q})
$$

Let us look in more detail at this transformation and some of its properties. The transformation can be split up in to components, namely the transformation from $\mathbf{q}$ to $\phi: \phi=T_{\phi}(\mathbf{q})$ and the transformation from $\mathbf{q}$ to $\mathbf{r}$ $\mathbf{r}=T_{r}(\mathbf{q})$, thus

$$
\mathbb{T}=\left[\begin{array}{c}
T_{\phi} \\
T_{r}
\end{array}\right]
$$

Since we are not only interested in the transformation of the state variables but also in the transformation of the dynamics under this transform let us investigate the derivatives of the transformed coordinates, we do this exemplary on $\phi$ since it works the same way for all state variables.

$$
\begin{gathered}
\phi=T_{\phi}(\mathbf{q}) \\
\Rightarrow \dot{\phi}=\frac{d T_{\phi}(\mathbf{q})}{d t}=\frac{\partial T_{\phi}(\mathbf{q})}{\partial \mathbf{q}} \dot{\mathbf{q}} \\
=\frac{\partial T_{\phi}(\mathbf{q})}{\partial \mathbf{q}} \mathbf{F}(\mathbf{q})
\end{gathered}
$$

We see that $T_{\phi}$ is intrinsically defined (by the fact that Eq. 7 has to be equivalent to $\omega$, being a constant) but there is some freedom in the choice of $T_{r}$. To remove this ambiguity we define the behavior of an oscillator in the canonical PRCS as

$$
\begin{gathered}
\dot{\phi}=\omega \\
\dot{\mathbf{r}}=1-\mathbf{r}
\end{gathered}
$$

This is somewhat arbitrary but the choice will become clear later in the discussion of the relationship of PRCS and QCS. At this place its choice is already partially motivated by Eq. 9 representing the simplest dynamical system with stable, non-zero fixed point behavior where the fixed point is $\mathbf{r}=1$.
The inverse of $\mathbb{T}, \mathbb{T}^{-1}$, transforms the system from the PRCS into the QCS. This means by designing $\mathbb{T}^{-1}$ the PRCS can be mapped into any type of oscillator.

Example: Consider the transform of $r$ into $r^{\prime}$, given by $r^{\prime}=(r-1)^{g}+r_{0}$. This transforms the canonical oscillator (Eqs $8-9)$ into

$$
\left[\dot{\phi}, \dot{r}^{\prime}\right]^{T}=\left[\omega,-g\left(r^{\prime}-r_{0}\right)\right]^{T}
$$

We can now transform this system further by applying the well known transformation from a Polar coordinate system into the Cartesian coordinate system: $q_{1}=$ $r^{\prime} \cos \phi, q_{2}=r^{\prime} \sin \phi$. By this transform we yield the following system

$$
\left[\begin{array}{l}
\dot{q}_{1} \\
\dot{q}_{2}
\end{array}\right]=\left[\begin{array}{l}
g\left(\frac{r_{0}}{\sqrt{q_{1}^{2}+q_{2}^{2}}}-1\right. \\
g\left(\frac{r_{0}}{\sqrt{q_{1}^{2}+q_{2}^{2}}}-1\right) q_{1}-q_{2} \omega \\
q_{2}+q_{1} \omega
\end{array}\right]
$$

We have thus transformed the canonical oscillator into a phase oscillator in a Cartesian coordinate system. The radius and convergence rate of the oscillator can be controlled by $r_{0}$ and $g$ respectively.

While here we can express the transform $\mathbb{T}^{-} 1$ and its inverse by rather simple mathematical expressions this is usually not possible. Even more, the transform might often not be expressible in a closed analytical form.

\subsection{Graphical assessment of $\mathbb{T}$}

We will now discuss a graphical way of assessing relationship of PRCS and QCS, i.e. $\mathbb{T}$ for a given oscillator. For this the notion of time becomes important, i.e. at what velocity the phase point moves through the phase space. What specifies the equivalence of a point in QCS and a point in PRCS? If for time $t_{0} \mathbf{q}\left(t_{0}\right)=\mathbb{T}^{-1}\left(\left[\phi\left(t_{0}\right), \mathbf{r}\left(t_{0}\right)\right)\right.$, then for all time $\mathbf{q}(t)=\mathbb{T}^{-1}([\phi(t), \mathbf{r}(t))$. This means if we could find a way of comparing the development of the two points in both coordinate systems at regular intervals and repeat this for different initial conditions we would get an idea of $\mathbb{T}$.

The activity of the oscillator can be plotted in the QCS (phase portrait) in which the limit cycle will show up as a closed curve. But, the information about the phase velocity is lost. So even that we know that for example the limit cycle in the QCS corresponds to the limit cycle in the PRCS, we do not know which point on the limit cycle in one coordinate system corresponds to which point in the other one. While in PRCS the phase moves along the limit cycle with constant velocity $\omega$, in general, for an arbitrary oscillator $\mathbf{F}$ in the QCS, the phase point will not move along the limit cycle in the phase space with a constant velocity. Thus, the phase does not correspond to the simple "position" on this curve (i.e. an infinitesimal part of the curve does not correspond to the same infinitesimal of $\phi, d \mathbf{q} \neq d \phi)$. In 
order to investigate on this relationship, we could plot points with always equal $\Delta \phi$.

However, the phase is only uniquely defined on the limit cycle, but we would like to get a global idea of the transformation. We can generalize the notion of a phase outside the limit cycle by the concept of isochrones. We follow the definition of (Pikovsky et al., 2001) and generalize the phase by help of the cycle time $T$, i.e. the mapping $\mathbf{q}(t) \rightarrow \mathbf{q}(t+T)$.

Definition 5 Isochrones - The set of points being invariant under the mapping $\mathbf{q}(t) \rightarrow \mathbf{q}(t+T)$ and crossing the limit cycle at $\mathbf{q}^{\star}$ (i.e. $\mathbf{q}^{\star}$ is a fixed point of the mapping) is called an isochrone through $\mathbf{q}^{\star}$.

In other words, all points of the phase space which converge to having the same phase on the limit cycle form an isochrone.

If we plot isochrones for every $\Delta \phi=$ const they show the relationship of the phase with the geometry of the system in the QCS (cf. Fig 2). Where they are tightly spaced the phase point moves slowly, thus values of different phase are tighter spaced. If they are equally spaced on the limit cycle, the lengths of an arc of the limit cycle is proportional to a $\Delta \phi\left(d \phi=\frac{1}{S} 2 \pi d s\right.$, where $s$ is the arclength and $S$ the total length of the limit cycle). If the isochrones are straight (as in Fig. 2b) this means that the DE for $\phi$ and $\mathbf{r}$ are decoupled, and the transformation $\mathbb{T}$ corresponds to a transformation from Cartesian to Polar coordinates.

Now, the isochrones give us an idea how $\phi$ is embedded into QCS. But in order to complete our picture of the transformation $\mathbb{T}$ we need to get an idea of how $\mathbf{r}$ is embedded into this coordinate system.

For this we define the Radius-Isochrones, which will give us an idea on how $\mathbf{r}$ evolves over time.

Definition 6 Radius-Isochrones - The set of points $\mathbf{q}(t)$ satisfying $\operatorname{dist}\left(\mathbf{q}(t+\Delta t), \mathbf{q}_{\infty}\right)=\epsilon$, are called a Radius-Isochrone with $\Delta t$.

Function dist denotes the distance between the point $\mathbf{q}$ and the limit cycle. Intuitively this means all points which converge to the limit cycle in the same time form a radius-isochrone. We see that the definition of the radiusisochrones implies a distance measure from the limit cycle. This distance can be defined in different ways. We use the perpendicular direction to the limit cycle and consider as converged to the limit cycle when it enters the "tube" of radius $\epsilon \ll 1$ around the limit cycle.

We can use the isochrones and the radius-isochrones to get an idea how the abstract phase-radius oscillator is embedded into the coordinate system for $\mathbf{q}$. The time to get from one isochrone to the next is constant, the time from one radius-isochrones to the next is also constant. The radius-isochrones gives information about the rate of convergence, tightly space radius-isochrones mean a slow convergence to the limit cycle, widely spaced mean fast convergence (see Fig. 2 and its description).
If we plot isochrones equally spaced in $\phi$ and radiusisochrones for equally spaced $\Delta t$ for the oscillator in the canonical PRCS (Eqs. 8-9) we get a rectangular grid as seen in Fig. 2a with equally spaced vertical lines and exponentially spaced horizontal lines. This means whenever we see those characteristics in an oscillator we know it behaves like the canonical oscillator (i.e. exponential convergence towards the limit cycle and isochrone behavior).

\subsection{Limit cycles under perturbations}

Considering the fact that we can transform every oscillator into a PRCS, it gets clear that we can consider all unperturbed oscillators are equivalent up to a transformation $\mathbb{T}$. They only differ in the way how a (physical) input to the system affects its dynamics, i.e. how the input output space is related to PRCS. So far we considered the autonomous, i.e. unperturbed, oscillator. However, the advantage of the use of oscillators (e.g. vs. function based approaches) becomes only effective when using them to be coupled to perturbations. This can mean the oscillator is coupled to some external in- and output or to other oscillators.

If the input and outputs are formulated in a QCS then, in respect to their influence on the limit cycle, they undergo the same transformation $\mathbb{T}$. We thus have to investigate limit cycle systems under perturbations. Therefore, we have to consider the single oscillator as non-autonomous system,

$$
\dot{\mathbf{q}}=\mathbf{F}(\mathbf{q}, \mathbf{p}(t))
$$

It is important to stress, that the perturbation can theoretically have any arbitrary functional form, and the perturbation is also not limited on acting on the first state variable only. However, a discussion of the different types of perturbations is out of the scope of this article. We will focus on an often used form of perturbation, the additive perturbation

$$
\dot{\mathbf{q}}=\mathbf{F}(\mathbf{q})+\mathbf{p}(t)
$$

We will however realize that the additive form in a QCS transforms into a more complicated functional form in the PRCS. The oscillator in the PRCS becomes

$$
\begin{gathered}
\dot{\phi}=\omega+p_{\phi} \\
\dot{\mathbf{r}}=\mathbf{F}_{r}(\mathbf{r}, \phi)+\mathbf{p}_{\mathbf{r}}
\end{gathered}
$$

$p_{\phi}$ is the component of the perturbation acting on the phase and $\mathbf{p}_{\mathbf{r}}$ is the component of the perturbation acting in direction of the radius.

Expressed by help of the transformation $\mathbb{T}$ we yield for the additive case (i.e. Eq. 13)

$$
\Rightarrow \dot{\phi}=\frac{\partial T_{\phi}(\mathbf{q})}{\partial \mathbf{q}} \mathbf{F}(\mathbf{q})+\frac{\partial T_{\phi}(\mathbf{q})}{\partial \mathbf{q}} \mathbf{p}
$$



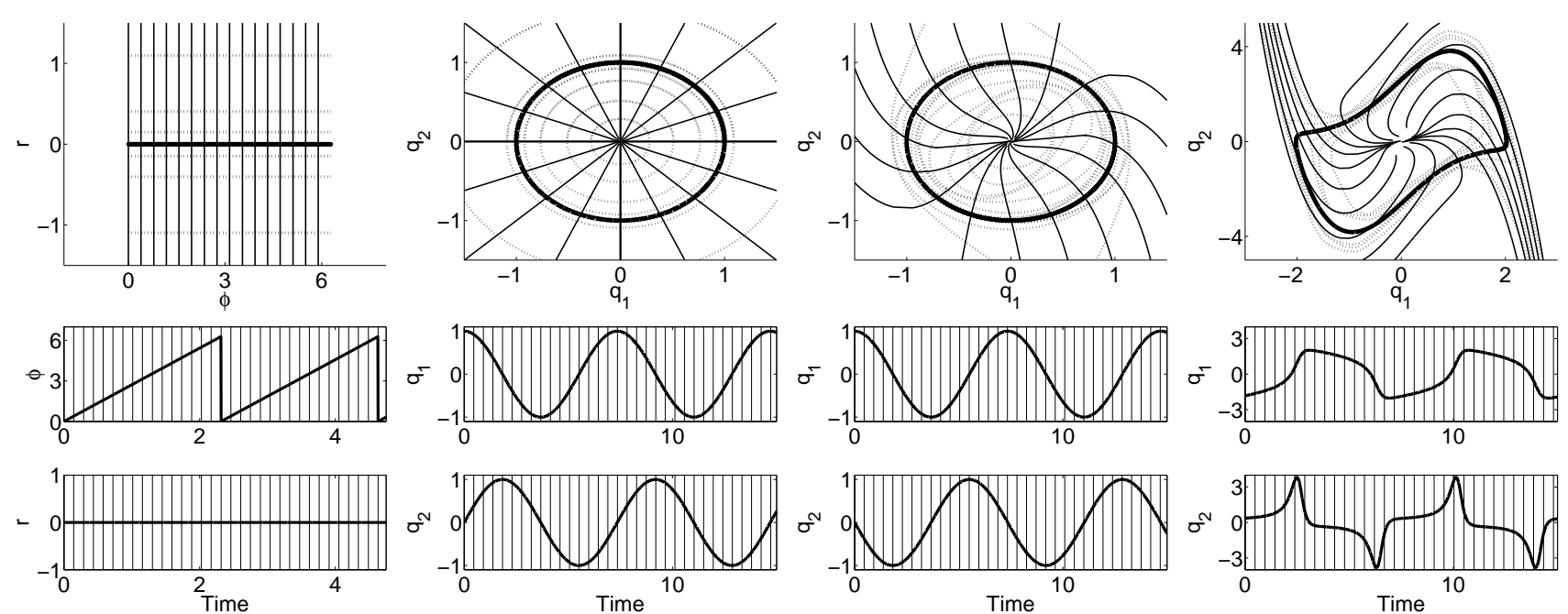

Fig. 2 Illustrating the transformation $\mathbb{T}$, by help of the isochrones and the radius-isochrones. For each oscillator 16 equally spaced isochrones are used and a varying number of radius-isochrones with a given $\Delta t$ are plotted in the phase portrait (upper panels) and below the time series are shown. The fine vertical lines indicate the isochrones (only indicating the temporal position on the time series.) We see while they are always equally-spaced in the time series plot, in the phase plot this is not necessarily the case. a) The phase plot of the canonical oscillator in the phase-radius coordinate system PRCS (Eqs. 3-4). The isochrones form straight and equidistant vertical lines. The radius-isochrones $(\Delta t=[5,6,7] s)$ form exponentially spaced straight horizontal lines. b) The Hopf oscillator (Eqs.30-31). The isochrones form straight rays at equal angles, which reflects the polar interpretation of $\phi$ in the transformation. The radius-isochrones $(\Delta t=[0.7,1.4,2.1] s$ outside of the LC and $\Delta t=[0.7,1.4,2.1,3.5] \mathrm{s}$ inside) reflect the 3 th order convergence behavior of the radius. In the time-series we see the harmonic nature of the limit cycle reflected. c) The Energy oscillator (Eqs. 22-23). Due to the appearance of the nonlinear energy term only in the first ODE the system looses its circular symmetry. The isochrones and the radiusisochrones $(\Delta t=[2,2.4,2.8,3.2] s$ outside of the $\mathrm{LC}$ and $\Delta t=[2,2.4, \ldots, 5.2] \mathrm{s}$ inside $)$ get a characteristic deformation. $\mathrm{d})$ van der Pol Oscillator (Eqs.42-43). This is a strongly nonlinear oscillator. This fact is reflected in the strong deformation of the isochrones. The strong deformation of the radius-isochrones $\Delta t=[0.3,0.6,0.9] s$ away from the limit cycle in the upper left and lower right corner of the figure indicates the rapid convergence of the system in that region. It is immediately visible that the transformation from this QCS to the PRCS is a very complicated one.

On the limit cycle $\mathbf{e}_{\phi}=\mathbb{T}(\mathbf{q})$, thus the relationship between the PRCS and the QCS coordinate system is the determinant for the effect of the perturbations to the oscillator.

Let us give a geometric intuition, which lets us easily derive $p_{\phi}$, without relying on the transformation $\mathbb{T}$. But, this derivation is only valid on the limit cycle, while above transforms are more general.

To arrive at this, it is important to realize that every perturbation has a direction in the phase space. Consider a pulse like additive input, i.e. a infinitely short input at time $t_{p}$ The perturbation will bring the phase point away from the limit cycle. The stability properties will bring it back to the limit cycle, but on another position, relative to the unperturbed system. Thus the phase of the system $\phi_{\left(t_{p}+\right)}$ is not the same as before the perturbation $\phi_{\left(t_{p}-\right)}$, the phase is reset hence the term "phase resetting" (cf. Fig. 3).

Thus, for small perturbations the effect that remains in the system is the effect of the perturbation in direction of the phase $\mathbf{e}_{\phi}$, this is the direction tangential to the limit cycle or equivalently the direction $\dot{\mathbf{q}}$ :

$$
\mathbf{e}_{\phi}=\frac{\dot{\mathbf{q}}}{|\dot{\mathbf{q}}|}
$$

Therefore, the effective perturbation on the phase is

$$
p_{\phi}=\mathbf{p} \cdot \mathbf{e}_{\phi}
$$

The derivative of the phase becomes

$$
\dot{\phi}=\omega_{0}+\mathbf{p} \cdot \mathbf{e}_{\phi}
$$

So we found the sensitivity of the phase on perturbations:

$$
S_{p}(\mathbf{p}) \doteq \frac{p_{\phi}}{|\mathbf{p}|}=\frac{\mathbf{p}}{|\mathbf{p}|} \cdot \mathbf{e}_{\phi}=\frac{\mathbf{p}}{|\mathbf{p}|} \cdot \frac{\dot{\mathbf{q}}}{|\dot{\mathbf{q}}|}
$$

This means that depending on the state of the oscillator, the same perturbation can have a different influence, at one stage it can speed the oscillator slightly up at the other state slow it down. If the sum of this acceleration or de-acceleration is non-zero this leads to entrainment effects.

The sensitivity of the phase to perturbations is summarized in the phase-reset curve (PRC) and its generalization the phase sensitivity (PS). The PRC is a function which describes the effect of a unitary pulse like perturbation as a function of the phase of the oscillator it arrives at. In other words it tells how much the phase is shifted by that perturbation.

The phase sensitivity generalizes this idea as it does not restrict to a single pulse like perturbation per cycle, but it is an "instantaneous" description of the effect of perturbations. Due to its importance in the discussion 


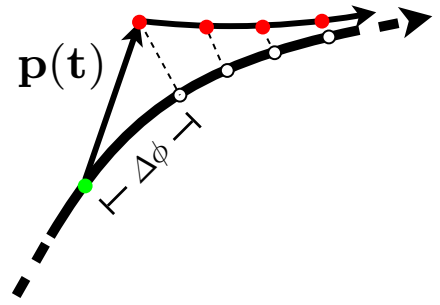

Fig. 3 Effect of a small pulse like perturbation on the limit cycle. The perturbation $\mathbf{p}(\mathbf{t})$ arrives when the phase point is at the position marked by a the green dot. The phase point is then pushed back to the limit cycle by the stability properties of the system, i.e. it approaches asymptotically the limit cycle. It however retains a phase difference $(\Delta \phi)$ in comparison with the unperturbed reference system. The phase difference can be of different amplitude and sign depending on the direction of the perturbation and the state the system currently is in when the perturbation arrives. Understanding this fact is key to understanding synchronization phenomena.

about influence of perturbation on oscillators a lot of research has been done mainly on PRC but also on PS, see (Pikovsky et al., 2001) and references therein. For an example of the derivation phase locking with the presented tools see (Buchli \& Ijspeert, 2004a).

It can be difficult or impossible to get analytical form of the PRC or PS. However, with the directional idea introduced in Eq. 20 it can be estimated from numerical integration. It can also be measured to a certain extent in real-world systems. We will come back to the role of the PRC/PS when discussing the design of entrainment effects in Section 5.

Since the frequency of the oscillator corresponds to the rate of change of the phase we see that in a perturbed oscillator the observed frequency is not necessarily the same anymore:

Definition 7 The instantaneous frequency $\Omega_{i}$ is de-
fined as the momentary rate of change of the phase
\[ \Omega_{i}=\dot{\phi} \]

While for the autonomous oscillator the instantaneous frequency is equal the intrinsic frequency and constant ( $\Omega_{i}=\omega=$ const $)$, in the perturbed oscillator the instantaneous frequency is not equal the intrinsic frequency $\left(\Omega_{i} \neq \omega\right)$ and is also a function of time $\left(\Omega_{i}=f(t)\right)$. $\Omega_{i}$ is the frequency which will be observed or measured at any given time (e.g. by methods like windowed FFT, spectrograms or wavelets). $\omega$ is the parameter, while $\Omega_{i}$ is a variable which can be decoupled from $\omega$ by a perturbation.

Remembering the relation $\Omega_{i}=\dot{\phi}$, this also means that the observed frequency is not the same as the intrinsic frequency: if we have phase locking (i.e. when the difference between the phase of the oscillator and the phase of the perturbation remains bounded) the observed frequency will be the frequency of the perturbation. Consider as an example two mutually connected oscillators with intrinsic frequencies $\omega_{1,2}$ which are different but close enough to have mutual entrainment. The observed frequency $\Omega_{i}$ will be the same for both oscillators, but different from the two intrinsic frequencies, i.e. $\Omega_{i} \neq \omega_{1,2}$ (it will be in between the two).

Thus, it can be said that the oscillator gets entrained by the perturbation it could be said that it adapts, but this change is only temporary, i.e. reactive. If the input is switched off the system immediately returns to its intrinsic dynamics, there is no memory of the input, no lasting change to the dynamics.

Further, there can be a influence on the radius by the perturbation which can also be exploited. The stability directions here are however less special so this usually reduces to quite standard treatment of ODEs with fixed points under perturbation. Note that however the behavior of the radius under perturbation can still be difficult, especially when there is a strong deformation of the radius-isochrones in the QCS (e.g. as for the van der Pol, cf. Fig. 2). Such a deformation means that the convergence behavior is very non-uniform and a perturbation has a completely different effect on $\mathbf{r}$ depending where it arrives.

Thus, summarizing the findings of this section, it must be realized that for the design it is important to know the stability properties and the effect of perturbations in the coordinate systems of the stability directions. Simply said, if we want to change radius only, then we need to act perpendicular the limit cycle, i.e. move the point on the same isochrone, if we want to affect the phase only we need to move the phase point tangential to the limit cycle, to move on the radius isochrones.

\section{The design space}

We realize that the oscillator can be completely reduced to the phase radius coordinate system and the inputs can be formulated in that system. We can then possibly use the transformation $\mathbb{T}^{-1}$ into a given interpretation coordinate system to talk about the oscillator in a physically or conceptually more meaningful coordinate system. So all design choices will deal in one way or the other with investigating what the effects of perturbation in the phase radius coordinate system (PRCS) are (and then possibly backwards via $\mathbb{T}$, what the this means for the behavior in the QCS).

We are now at a stage where we can discuss what can be designed in an oscillator. There are three basic ways how an oscillator and its in and output can be defined:

1. Chose an $O D E$ system $\mathbf{F}(\mathbf{q}, \rho)$ and functional form of in and output, i.e. $\mathbf{F}(\mathbf{q}, \rho) \rightarrow \mathbf{F}(\mathbf{q}, \rho, \mathbf{p})$. 
Then we need to work out the relation of $\mathbf{F}$ to $[\dot{\phi}, \dot{\mathbf{r}}]$, i.e. we have to work out $\mathbb{T}$ (or parts of it), the transformation is implicitly specified.

Here we need to able to convince ourselves either analytically or at least numerically that $\mathbf{F}(\mathbf{q}, \rho)$ represents indeed a structurally stable oscillator. Then we are sure about the existence of the phase radius coordinate system and the stability properties that have been discussed.

Example:

$$
\begin{aligned}
& \dot{q}_{1}=-\alpha \frac{q_{1}^{2}+q_{2}^{2}-E}{E} q_{1}-q_{2}+p_{x}(t) \\
& \dot{q}_{2}=q_{1}
\end{aligned}
$$

This oscillator's steady state solution is $\mathbf{q}_{\infty}=\left[\sqrt{E} \cos \left(t+t_{0}\right), \sqrt{E} \sin \left(t+t_{0}\right)\right]$. Structural stability is most easily shown by transforming into polar coordinates and showing that the ODE for the radius has a stable fixed point. We can then show with rather straightforward analysis that in stationary regime:

$$
\dot{\phi}=\omega+\sin (\phi) p_{x}(t)
$$

2. Use an ideal phase or phase radius oscillator and given input and output directly in this coordinate system, the transformation is thus implicitly specified by this choice.

Note that the transformation does not necessarily have to be fully specified, e.g. if only a scalar output is needed it suffices to define a part of the transformation.

Example: Thus, we choose a phase oscillator and add a nonlinearity in the input $\dot{\phi}=\omega+\sin (\phi) p(t)$ will synchronize on $p(t)$ if frequencies are close. The output is chosen to be $o=\sin (\phi)$.

3. Specify input in QCS and specify $\mathbb{T}^{-1}$ explicitly. Example: Choose $\mathbf{q}=\left[q_{1}, q_{2}\right]$, a Cartesian coordinate system, and $\mathbb{T}$ as the transformation into a polar coordinate system, i.e. $T_{\phi}=\arctan \frac{q_{1}}{q_{2}}(\arctan$ denotes the four-quadrant arcus-tangent), $T_{\mathbf{r}}=\sqrt{q_{1}^{2}+q_{2}^{2}}$. The input acts on the first state variable only: $\mathbf{p}=$ $\left[p_{1}, 0\right]$. We can split up the relevant term from Eq. 16 in the following way

$$
\frac{\partial T_{\phi}(\mathbf{q})}{\partial \mathbf{q}}=\left[T_{\partial q_{1}}, T_{\partial q_{2}}\right]^{T}
$$

Since $\mathbf{p}=\left[p_{q_{1}}, 0\right]^{T}$, we can write

$$
p_{\phi}=\left[T_{\partial q_{1}}, T_{\partial q_{2}}\right]^{T} \cdot\left[p_{q_{1}}, 0\right]^{T}=T_{\partial q_{1}} p_{q_{1}}
$$

Using $\phi=\arctan \frac{q_{1}}{q_{2}}$

$$
\begin{gathered}
T_{\partial q_{1}}=\frac{d}{d q_{1}} \arctan \frac{q_{1}}{q_{2}}=\frac{1}{1+\left(\frac{q_{2}}{q_{1}}\right)^{2}} \frac{-q_{2}}{q_{1}^{2}} \\
=-\frac{r \sin \phi}{r}=\sin \phi \\
\Rightarrow p_{\phi}=-\sin \phi p_{q_{1}}
\end{gathered}
$$

As one can see, the three examples are equivalent in their phase behavior. We also see that it is often not needed to have a full knowledge of the theoretical transformation $\mathbb{T}$.

In large parts of the literature we see method 1 employed. Often with some complicated $\mathbf{F}$ out of the modeling literature. Immediately we realize that for engineering this is often not the best choice.

By help of the introduction of the stability directions and the geometrical aspects of interpretation of the phase-radius coordinate system (i.e. $\mathbb{T}$ ), we can identify two somewhat orthogonal design axes: (1) Timing: Design that influences the phase of the oscillator, here we have (a) influence on (relative) phase, (b) instantaneous frequency, and (c) average frequency. (2) Design that influences the geometry of the oscillator. To name here are (a) influences on $r$ directly and (b) the design of $\mathbb{T}^{-1}$, i.e. output filter. This is a very interesting and important result, since orthogonal design axis are extremely helpful for engineering tasks. It means we can decouple the influence of parameters on the outcome. In other words, by choosing a more suitable coordinate system, those influences get decoupled while in the original coordinate system they are not.

To detail this more, in the following we address some common design goals and how they translate into properties which the system has to exhibit (summary in Table 2).

1. Specification: Unperturbed average frequency: $\bar{\Omega}_{i}=$ $\omega$. Average observed frequency should be equal to the intrinsic frequency $\omega$.

Required Property: $\int_{0}^{2 \pi} p_{\phi}=0$

We need the effect on the phase to have in average zero effect.

2. Specification: Frequency locking with an external signal of frequency $\omega_{F}$ i.e. $\bar{\Omega}_{i}=\omega_{F}$

Required Property: $\frac{1}{2 \pi} \int_{0}^{2 \pi} p_{\phi}=\omega_{F}-\omega\left(=\omega_{d}\right)$ if $\Omega_{i}=$ $\omega_{F}$, in differential terms: show that $\phi_{d}=\phi-\phi_{F}$ is bounded $\int_{0}^{2 \pi} \dot{\phi}_{d}=\int_{0}^{2 \pi} p_{\phi}+\omega_{d}=0$ the perturbation in the phase needs in average to make up for the differences between the intrinsic frequency and the frequency of the perturbation.

3. Specification: Phase locking with arbitrary phase lag: $\phi_{d}=\Phi_{r}$.

Required Property: $\dot{\phi}_{d}\left(\Phi_{r}\right)=p_{\phi}\left(\Phi_{r}\right)+\omega_{d}=0$ and $\left.\frac{d}{d \phi} \dot{\phi}_{d}\right|_{\Phi_{r}}<0$. The DE for $\phi_{d}$ needs a stable fixed point at $\Phi_{r}$. This is achieved when the perturbation at every instant cancels for the differences between the intrinsic frequency and the frequency of the perturbation. Note, phase locking implies frequency locking.

4. Specification: Arbitrary instantaneous frequency: $\dot{\phi}(t)=$ $\Omega_{r}(t)$.

Required Property: $p_{\phi}=\Omega_{r}(t)-\omega$. 


\begin{tabular}{|c|c|c|}
\hline & Specification & Required Property \\
\hline 1) & Unperturbed avg. frequency: $\bar{\Omega}_{i}=\omega$ & $\int_{0}^{2 \pi} p_{\phi}=0$ \\
\hline 2) & Frequency locking: $\bar{\Omega}_{i}=\omega_{F}$ & $\begin{array}{l}\frac{1}{2 \pi} \int_{0}^{2 \pi} p_{\phi}=\omega_{F}-\omega\left(=-\omega_{d}\right) \text { if } \Omega_{i}=\omega_{F}, \text { in differential } \\
\text { terms: show that } \phi_{d}=\phi-\phi_{F} \text { is bounded } \int_{0}^{2 \pi} \dot{\phi}_{d}= \\
\int_{0}^{2 \pi} p_{\phi}+\omega_{d}=0\end{array}$ \\
\hline 3) & Phase locking: $\phi_{d}=\Phi_{r}$ & $\begin{array}{l}\dot{\phi}_{d}\left(\Phi_{r}\right)=p_{\phi}\left(\Phi_{r}\right)+\omega_{d}=0 \text { and } \frac{d}{d \phi} \dot{\phi}_{d}\left(\Phi_{r}\right)<0 \text { (phase } \\
\text { locking implies frequency locking). }\end{array}$ \\
\hline 4) & Spec. instantaneous frequency: $\dot{\phi}(t)=\Omega_{r}(t)$ & $p_{\phi}=\Omega_{r}(t)-\omega$ \\
\hline 5) & $\begin{array}{l}\text { Arbitrary output signal shape: } x(\phi)=x_{r}(\phi) \text { or } x(\mathbf{q})= \\
x_{r}(\mathbf{q})\end{array}$ & Appropriate function, i.e. filter \\
\hline 6) & Arbitrary form of LC in QCS: $\mathbf{q}_{\infty}=\mathbf{q}_{r e f}(t)$ & $\begin{array}{l}\text { stability directions i.e. } \frac{\mathbf{F}\left(\mathbf{q}_{r e f}\right)}{\mid \mathbf{F}\left(\mathbf{q}_{r e f} \mid\right.}=\frac{D\left(\mathbf{q}_{r e f}\right)}{\left|D\left(\mathbf{q}_{r e f}\right)\right|} \text { and } \\
\frac{\partial \mathbf{n}\left(\mathbf{q}_{r e f}\right)}{\partial \mathbf{n}}<1\end{array}$ \\
\hline
\end{tabular}

Table 2 Common design goals and the required properties of the oscillator.

5. Specification: Arbitrary T-periodic output signal shape Required Property: An appropriate output function or dynamical system has to be found, i.e. this leads to filter design, or the design of $\mathbb{T}^{-1}$.

6. Specification: Arbitrary form of limit cycle in QCS, i.e. $\mathbf{q}=\mathbf{r}(t)$ - one or several state variables should follow a reference trajectory. This means we want a general form of the limit cycle: We have a closed curve in QCS which should be the limit cycle. Required Property: We need thus to design the stability directions to be tangent, i.e. on the curve the flow has to have the direction of the tangent. Normal to the curve the flow has to be stable. If $D($.$) denotes the$ tangential and $\mathbf{n}($.$) the normal direction to a curve,$ we require (i) flow tangential to reference trajectory $\frac{\mathbf{F}(\mathbf{r}(t))}{\mid \mathbf{F}(\mathbf{r}(t) \mid}=\frac{D(\mathbf{r}(t))}{|D(\mathbf{r}(t))|}$ and (ii) contracting perpendicular to the limit-cycle $\frac{\partial \mathbf{n}(\mathbf{r}(t))}{\partial \mathbf{n}}<1$.

Remember that if we decide to work with a QCS, the the perturbation on the phase is $p_{\phi}=f(\mathbb{T})$, thus the properties to be satisfied to meet above design goals contain the relationship between a chosen coordinate system (QCS) and the PRCS.

The properties listed above will not necessarily allow a directed design without further assumptions and simplifications as they can lead to very difficult expressions. In the second part of the article we will address how some of the above properties have been designed in previous work.

\subsection{Classes of Limit Cycles}

Here we list a few important classes of oscillators, with properties in terms of the above discussed topics. They are loosely ordered from "simple" to more "complicated" (in the sense of $\mathbb{T}$ ). As we have seen above, the classification only makes sense for a given coordinate system. We present them in the coordinate system in which they are usually used and additive input is used.
Phase oscillator - The probably simplest type of oscillator, where the radius is completely neglected, only the phase is retained. The phase oscillator is a linear system defined on the circle $\mathbb{S}^{1}$ instead of the Euclidean space $\mathbb{R}^{1}$ which implies a closeness of the solution, and with that the system fulfills our definition of an oscillator.

Properties The phase oscillator is essentially a first order linear differential equation, the nonlinearity lies in the interpretation of the phase modulo $2 \pi$ and input/output relation. It is the most abstract oscillator. An important assumption for applicability of phase oscillator is that the limit cycle is strongly damped, i.e. that the phase point is always on the limit cycle (or very close).

The output signal shape can not change based on input (other than direct functional coupling).

References: The phase oscillator were and are the "work-horse" to work on synchronization effects, e.g. the Kuramoto oscillators (Kuramoto, 1984; Strogatz, 2000).

Equations

$$
\dot{\phi}=\omega+p(F(t), \phi)
$$
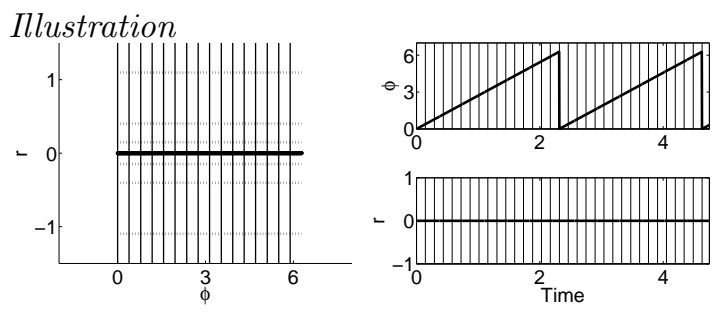

\section{Isochronous oscillator}

Properties The isochronous oscillators has straight isochrones, i.e. they are perpendicular to the limit cycle.

Equations

In the simplest case, the isochronous oscillator is a linear differential equation system. However not defined on the Euclidean $\mathbb{R}^{D}$ space but on $\mathbb{S}^{1} \times \mathbb{R}^{D-1}$ $\dot{\phi}=\omega \bmod 2 \pi$

$\dot{\mathbf{r}}=f\left(\mathbf{r}_{0}, \mathbf{r}, \phi\right)$ 
Where $f\left(\mathbf{r}_{0}, \mathbf{r}\right)$ is DE with stable fixed point $\mathbf{r}_{0}$. The interpretation of $\phi$ modulo $2 \pi$, e.g. in Cartesian coordinates makes it an oscillator, e.g. the Hopf oscillator

$$
\dot{x}=\left(\mu-r^{2}\right) x+\omega y
$$$$
\dot{y}=\left(\mu-r^{2}\right) y-\omega x
$$
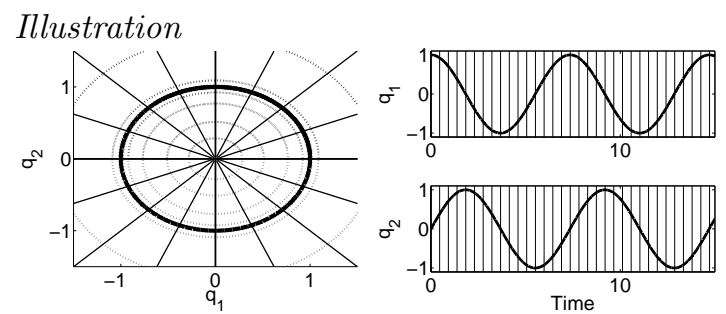

References The oscillator in (Buchli \& Ijspeert, 2004a) is isochronous.

Amplitude controlled phase oscillators (ACPO)

The ACPO is the extension of the phase oscillator with a radius. The radius is controlled by a differential equation with a fixed point attractor.

Properties The ACPO simple in the sense that the phase shows up as an explicit state variable. This often allows for analytical treatment (Buchli \& Ijspeert, 2004a). However, in order to achieve higher order locking the input needs to be generating to opportunities for these locking regimes, in the sense that it needs to generate higher order harmonics or subharmonics of the input. In contrast to the isochronous oscillator, the differential equation for the radius depends on the phase.

\section{Equations}

$\dot{\phi}=\omega$

$\dot{\mathbf{r}}=\mathbf{F}(\mathbf{r}, \phi)$

$f$ is a nonlinear function with stable fixed point. Illustration
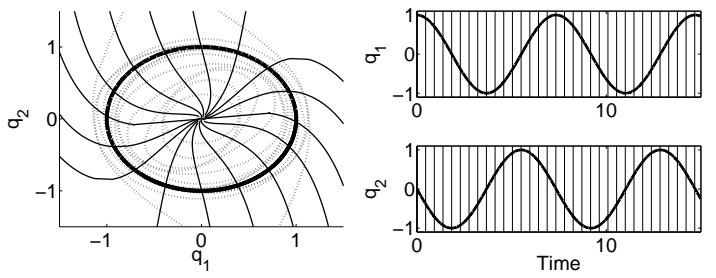

Harmonic oscillators - An oscillator with a harmonic limit cycle: i.e. stationary solution $\mathbf{q}_{\infty}(t)=$ $[r \cos (t), r \sin (t)]$. Note that this term conflicts with the common use to describe a linear second order system. Such a system is however not an oscillator after our definition as discussed above.

Properties Due to its harmonic limit cycle some analytical results on the PS/PRC are possible and thus closed form solutions for phase relationships and locking behavior can be derived.

Typical for harmonic oscillators is a possible description in Cartesian coordinates where the linear second order oscillatory system shows up with an addition of nonlinear terms stabilizing the radius.
Equations

$\dot{q}_{1}=\omega q_{2}+f_{1}(\mathbf{q})$

$\dot{q}_{2}=-\omega q_{1}+f_{2}(\mathbf{q})$

See Eqs. 22- 23 for a concrete example.

Illustration See illustrations for the isochronous oscillator and ACPO which are both harmonic oscillators. References The Hopf oscillator (Hopf, 1942) and the oscillators in (Ijspeert, Crespi, \& Cabelguen, 2005; Buchli \& Ijspeert, 2004a) are harmonic oscillators.

Piecewise Linear Systems - A system constructed of a set of linear systems of the same order, of which always one is active depending on conditions on the state variables.

Properties Rather straight forward to design and analyze (piece wise solution), can be problematic to simulate. Physical interpretation of switching effect is some fast effect.

Equations Consider the oscillator from (Taga, 1994)

$$
\begin{aligned}
& \dot{q}_{1}=-q_{1}-w \max \left(q_{3}, 0\right)-\beta q_{2}+1 \\
& \dot{q}_{2}=-q_{2}+\max \left(q_{1}, 0\right) \\
& \dot{q}_{3}=-q_{3}-w \max \left(q_{1}, 0\right)-\beta q_{4}+1 \\
& \dot{q}_{4}=-q_{4}+\max \left(q_{3}, 0\right)
\end{aligned}
$$

The system is switched whenever one of the state variables $q_{1,3}$ crosses 0 , so the above form is a short form to describe four different systems and oscillates for certain parameter values (e.g. $w=2.5, \beta=2$ )
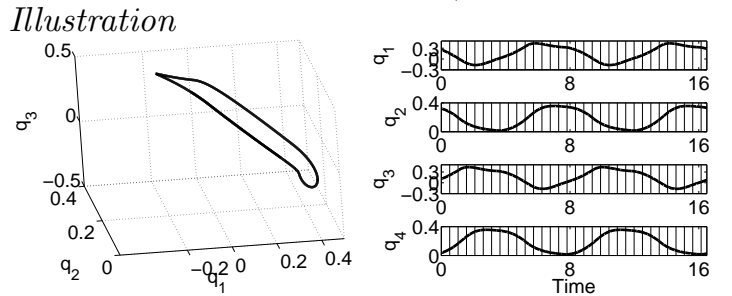

References The well known Matsuoka oscillators (Matsuoka, 1985), applications of the Matsuoka oscillator in (Taga, 1994; 22, 2003). Such systems are also known as switched linear system or hybrid systems.

Linear systems with reset - A linear, often second order system, which is reset if a variable passes a certain threshold. Those system are an approximation of the relaxation oscillators where we can consider the fast effect to be infinitely fast.

Properties In between resets they behave like a linear system which implies that they are analyzable under this condition, i.e. a partial tractability. Can be problematic for certain solver schemes due to the discontinuity in the ODEs introduced by the reset. Due to the fact that by the reset the whole semi-plane $q_{2}>1$ is reduced to a point $(\mathbf{q}=[0,1])$, the isochrones and radius-isochrones are identical except for the point on the limit cycle. Therefore, the transformation $\mathbb{T}$ is mathematically problematic. 
Equations From (Izhikevich, 2001)

$\dot{q}_{1}=b q_{1}-\omega q_{2}+I$

$\dot{q}_{2}=\omega q_{1}+b q_{2} \quad$ If $q_{2}>1,\left[q_{1}, q_{2}\right]=[0,1]$

where $b<0$ and $I$ are constants.

Illustration
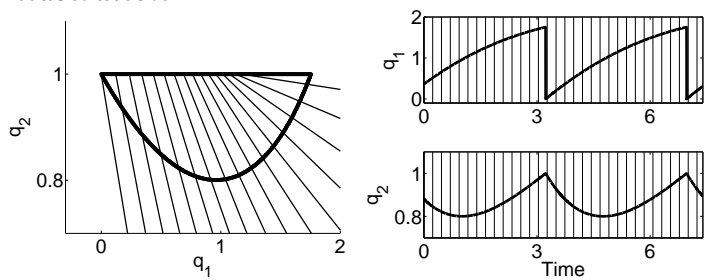

References The resonant and fire neurons in (Izhikevich, 2001).

Relaxation oscillators - Properties

In general no closed form solution, characteristic phase space with sharp corners, a fast/slow system i.e. two involved time scales which can often been related to physical mechanisms. Relaxation oscillators allow for fast phase locking due to their bent isochrones. Furthermore, they allow naturally higher order locking. Equations The van der Pol Oscillator

$$
\begin{aligned}
& \dot{q}_{1}=q_{2}+p(t) \\
& \dot{q}_{2}=\mu\left(p^{2}-q_{1}^{2}\right) q_{2}-\nu^{2} q_{1}
\end{aligned}
$$
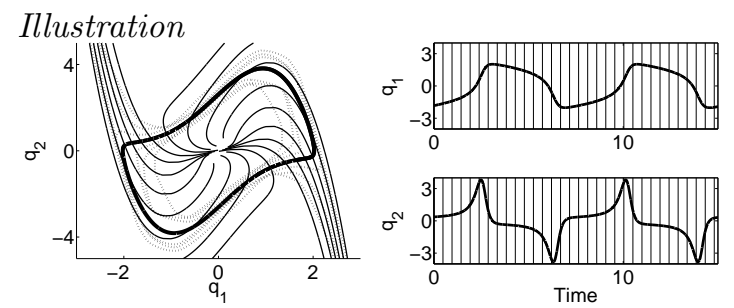

References The well known Hodgkin-Huxley (HH) model of the giant squid axon (Hodgkin \& Huxley, 1952) can exhibit relaxation type oscillatory activity, by a simplification of HH the Fitzhugh-Nagumo (FHN) Oscillator (FitzHugh, 1961) have been derived. The FHN is very closely related to the van der Pol Oscillator.

It is important to realize that a given oscillator can belong the several of the here presented classes (i.e. isochronous, harmonic, ACPO are not mutually exclusive).

\section{Design approaches}

Thus so far we have seen what can be designed. In this section we address the issue how the design goals can be achieved. Thus, as we have seen we need to find structure and parameterization of either $\mathbf{F}$ or $\mathbb{T}$. Those can be found in different ways, with off-line and online methods.

Off-line - Here the structure and parameters are found by some process before the systems is deployed. Once the system is working they remain fixed. There are different ways to find a suitable structure and parameterization of the ODEs:

(1) System can be designed by hand, by help of suitable mathematical tools. (2) By search/optimization (i.e. an algorithm outside of the dynamical system) (3) Dynamically shaped (i.e. the tuning is part of the dynamical system), but once deployed this process is frozen.

On-line adaptation

We could also imagine having some of the parameters changing over time as the system is deployed. This basically means that the parameters are not constant any more. They are turned in a certain sense into state variables as well. Again different approaches can be used: (1) By an algorithm which is outside of the dynamical system. Usually this includes the assessment/measurement of the some predefined quality of the system and an algorithm which tunes the parameters to achieve a better quality. (2) Dynamically Here the relevant parameters are turned into state variables and a dynamic law in form of ODEs has to be found that will tune the system into the required dynamics. This is very recent research with oscillators (early work (Ermentrout, 1991; Nishii, 1998, 1999), more recent (Buchli \& Ijspeert, 2004b; Righetti et al., 2006)). We only call such systems adaptive, since they combine the to be exploited dynamics and the adaptation process into a single dynamical system. This is also in line with the use of the term adaptive in the framework of adaptive control but is in contrast to the use of the term in many applications of oscillators.

We can thus change the properties as discussed before in Section 3: (1a,b,c,2a,b) and use the here described design approaches. In table 3 we list some of the literature in which oscillators are used either in modeling which had influence on robotic applications, are related to or are directly robotic applications. In the next section we will discuss some aspects of Table 3 in more detail.

\section{Design results: From reactive to adaptive oscillators}

Thus, we have come a long way in describing the basic characteristic of oscillators, how they lead to design specification and properties. Finally, we would like to discuss some aspects of the resulting system. The resulting systems can have different properties in terms how they react to the perturbation, how long information about the perturbation is retained. The oscillator can be purely reactive, i.e. the perturbation has only a short term, transient effect. Or the system can be adaptive. The system has a memory and the effect of the information stays possibly for infinite time. We note this classification in Table 3 in the first column by R/A. 
5.1 Reactive: Temporary entrainment and shape changes

The basic property of structurally stable dynamical systems can be exploited. In other words, the fact that their behavior is a combination of their intrinsic dynamics and external input. This means external inputs can partially modify or even "override" the autonomous behavior of the system (annihilate attractors/induce bifurcations). This can possibly be exploited for applications. The changes to the system are reactive in the sense that there is no lasting change in the system. If the input signal is switched off the system will immediately behave according to its original autonomous dynamics. In other words the parameter, i.e. intrinsic dynamics, stays constant. Memory effects can only be realized by the state of phase point and are transient and shortlived. In this category we can count all the exploitation of synchronization, phase resetting etc. (such as in (Ermentrout \& Kopell, 1994; Williamson, 1998; Ijspeert et al., 2005; Matsuoka, 1985, 1987; Schöner et al., 1990; Schöner \& Kelso, 1988; Taga, 1994, 1995b, 1995a; Buchli \& Ijspeert, 2004a; 22, 2003; Schöner \& Santos, 2001; Santos, 2003, 2004; Collins \& Richmond, 1994; Endo et al., 2005)). As an example, in case of phase locking the oscillator is matching its frequency to the frequency of the input. This is reactive since the frequency does not stay in the system. The system has no memory of the frequency. The very moment the input is switched off it rotates with the intrinsic frequency. The only remaining perturbation is a possible shifted phase compared to the hypothetically same but unperturbed oscillator. Thus the system is more reactive than adaptive (despite the use of the word in many contributions). One can argue that the above made distinction a reactive and an adaptive system is somewhat arbitrary, but often we can argue by separation of time scales. The "parameters" will usually evolve on time scales slower than the "state variables". This separation of time-scales is an important concept in physics and engineering to decide which variables are considered static and which dynamic (Crawford, 1991; Haken, 1983).

Entrainment, synchronization $\&$ phase locking As we have seen in the previous section, the limit cycle has very characteristic stability properties: It is marginally stable in the direction of rotation. This implies that a perturbation in this direction is not "forgotten" by the system, while the perturbation perpendicular, i.e. asymptotically stable direction, to the limit cycle are damped out.

We can exploit the synchronization properties of a limit cycle system to sightly modify the oscillators timing so that it works "in step" with some outside process. A meanwhile very common application in robotics is to exploit synchronization for legs to work in step with some sensorial input (e.g. touch sensors on the foot, cf. (22, 2003; Taga, 1994; Morimoto et al., 2006)).
An important aspect in the design of oscillator especially if they are coupled with others into networks is often the question how to design the phase relationship, i.e. with which phase lag the activity of the oscillator is synchronized with the perturbation. An interesting approach to desing specific phase lags is the to use contraction theory (Wang \& Slotine, 2005).

As we have seen important concepts for the designing are the phase response curve (PRC) (Pikovsky et al., 2001) or the more general concept of Phase sensitivity (PS) (Ermentrout \& Kleinfeld, 2001; Kramer, Herschel, \& Calo, 1984; Buchli \& Ijspeert, 2004a). PRC/PS can be derived in an analytic fashion only for some types of oscillators (i.e. harmonic oscillators, phase oscillators; thus they might be the oscillator of choice for this reason). Basically having a closed form of PRC/PS is equivalent in knowing the closed form of the limit cycle. However for other, non-tractable oscillators, we can measure the PRC/PS by numerical integration. The PRC and PS typically have zeros which means that a perturbation arriving when the oscillator is at this phase does not affect the phase. The phase of the oscillator will thus be shifted by a perturbation until it reaches this point and remain there (given it is a stable point). This means to design a certain phase relationship, we have to design either the PRC/PS of the system, i.e. its zeros or need a filter to the input that the desired phase relationship is attained (e.g. rotation of signal as in (Buchli \& Ijspeert, 2004a)).

In (Buchli \& Ijspeert, 2004a) a discussion about the choice of oscillators can be found an it is shown that the simpler oscillators allow for a good design of some of the properties of network of oscillators used as CPG.

A key requirement for synchronization is that the frequency of the oscillator needs to be close to the frequency of the input. If the differences between the frequencies is large the oscillator does not fully synchronize, it will only show a tendency to synchronization, an effect which is called phase slips (Pikovsky et al., 2001). In average the signals of the oscillators will drift relative to each other. A way around this problem is to make the frequency adaptive which will be discussed in the next subsection.

Another way of designing phase relationship in networks of coupled oscillators is by help of the theory of symmetry, where the phase pattern can be achieved without the single oscillator satisfying necessarily the correct properties in the PRC/PS (Golubitsky \& Stewart, 2006; Schöner et al., 1990; Righetti \& Ijspeert, 2006a). However, even along with this method the consideration of the properties of the single oscillator helps to design non-frustrated systems, i.e. systems where the individual behavior is in accordance with the global pattern, which settle down faster and are more stable. The synchronization properties can also be exploited to sequence and time actions as outlined in (Schöner \& Santos, 2001; Santos, 2003, 2004). 
Inter cycle timing Another desired property could be that the input signal should influence the instantaneous frequency without changing the average observed frequency. The key to such behavior is the property 1) in Table 2 and discussed before. While we are not aware of a contribution directly exploiting this characteristic it would be straight forward to impose an additional constraint on the oscillator used in (Righetti \& Ijspeert, 2006a) to satisfy this property.

Reactive shape changes Changes of radius are less frequently exploited than the entrainment effects. But of course due to the stability properties we can very well imagine an input which deflects the limit cycle by changing temporarily the fixed point for the radius. This translates in the outputs to have temporarily a larger or smaller amplitude. An example can be found in (Righetti \& Ijspeert, 2006b).

Shaping the dynamical system Another way of designing an oscillator with given output is to use a system which is a universal approximator and approximate the oscillator with this dynamical system. In (Ruiz et al., 1998; Galicki et al., 1999; Leistritz et al., 2002) the authors use recurrent neural networks to achieve arbitrary limit cycles. The disadvantage of this method is that it usually leads to a very high dimensional system of which the influence of the parameters can not easily be grasped.

An alternative way to changing the intrinsic dynamics of the oscillator is to shape its output into to some given form with filters. One possibility is to design the filter purely functional or to design a dynamical dynamical system which transforms the output (cf. linear filter). In (Ijspeert et al., 2002) the authors use Gaussian filters shaped by locally weighted learning. In (Zegers \& Sundareshan, 2003) the authors use Neural Networks as filters. The use of filters has the disadvantage that a discontinuous change in the parameters of the filter can lead to a discontinuous change in the output (i.e. one of the advantages of using dynamical systems is negated). In (Okada et al., 2003) shaping of arbitrary limit cycles is discussed by a direct design of the flow. In (Righetti \& Ijspeert, 2006b) the authors shape the limit cycle by help of a network of adaptive frequency oscillators.

\subsection{Adaptation: Lasting changes to the dynamics}

In this section we address lasting changes to the intrinsic dynamics, i.e adaptation. Such lasting changes could also be called called learning and in some communities this is the preferred term. We do use the terms as equivalent here.

As we have seen the limit cycle system is parameterized by a set of parameters $\rho$, which are usually kept constant. Adaptation means now that we find a law to change some or all of the parameters so that an adaptation goal is achieved. There are two conceptually different ways of achieving that, either the parameters are changed by an external process or algorithm, or the dynamical system itself gets enhanced with additional state variables and ODEs that represent the parameters and their evolution respectively. This means we have to find suitable differential equations $\dot{\rho}=\mathbf{F}_{\rho}(\mathbf{q}, \rho, t)$. This implies that the set of parameters reflects the state of the adaptation process, especially also after the system is halted. We can thus possibly also read out certain information about the system. This adaptation process constitutes a longer-term memory, in contrast to the reactive changes in the previous section which are forgotten and do not get remembered in the state of the parameters.

The first, algorithmic method, is from the methodological point of view simpler since standard optimization, learn and search techniques can be employed. In (Marbach \& Ijspeert, 2005) Powell's method is used to optimize the parameters of an oscillator network. Many other optimization methods could be used for similar tasks. The second, dynamic method, is more appealing from the conceptual point of view and leads to more efficient and robust solutions (cf. (Buchli et al., 2006)).

Dynamic adaptation of limit cycle systems is a more recent development and young field of research. There are some investigations on adaptation of parameters (frequency, others), e.g. in (Nishii, 1999; Large, 1994; Buchli \& Ijspeert, 2004b; Righetti et al., 2006).

An example is introduced in (Buchli \& Ijspeert, 2004b) and analyzed in detail in (Righetti et al., 2006) where a Hopf oscillator (Eqs. 30-31) is enhanced with a evolution law for the frequency $\omega$ in the following way

$$
\dot{\omega}=-k \frac{y}{\sqrt{x^{2}+y^{2}}} p(t)
$$

where $k$ is a coupling constant and $p(t)$ an additive perturbation to Eq. 30. This law allows the oscillator to adapt to the frequency of the perturbation $p(t)$. Such an additional law for the parameter $\omega$ endows the system with many very nice properties and can be exploited for different tasks such as adaptation to body dynamics (Buchli \& Ijspeert, 2004b; Buchli et al., 2005, 2006) or programmable CPGs (Righetti \& Ijspeert, 2006b).

As can be seen in Table 3, the column with the adaption is only sparsely populated. A lot of questions have to be answered and methodologies have to be found.

Adaptation can also be used in the design phase and then the adaptation process is frozen for the deployment phase (i.e. online vs off-line adaptation). This means the dynamics is adapted, then remains fixed for the application (learning/exploitation phase is distinguished) whereas in the first case the adaptation works continously. 


\section{Conclusion \& Discussion}

Discussion of design choice Oscillators have been used widely in robotics over the last few years with a lot of success, however a lack of abstraction often leads to suboptimal solutions for the given goal. These suboptimal choices arise due to a lack of abstraction of the concepts and/or a fixation on traditionally used oscillators.

For engineering of an application with oscillators we first of all have to get clear what feature of an oscillator is the important one for the task. In other words, we have to decide if its a generic feature of oscillators, of a class of oscillators, or if its unique to a certain oscillator/input/output.

We have to think about readouts, what information needs to be available, e.g. an oscillatory output signal with certain properties (e.g. harmonic), or does the phase of the oscillator have to be available as output? We can then think if one of the well known oscillator/coupling schemes which is suitable for the task at hand. Of course sometimes it can be helpful to take an existing oscillator and modify it to fit (cf. e.g. (Kay, Kelso, Saltzman, \& G., 1987; Righetti \& Ijspeert, 2006a))

Most of the time oscillators are used for their synchronization properties. Thus, we are interested in how the phase $\phi$ behaves over time. In addition influence on the radius can be exploited, but this is far less common. This means that for many applications the phase oscillator will a good and completely sufficient choice.

While it is often very convenient to use a phase oscillator we have to be careful with coupling which explicitely use the phase of the input signal (i.e. as it is often done in work on coupled phase oscillators). Because couplings that worked out this way do usually not generalize so easily to a general periodic signal of which the phase is not directly accessible. A way to bridge the gap and to investigate arbitrary periodic signals are Fourier series.

As always in engineering it is not possible to give rules that are valid for all cases, but important questions to guide the choice of oscillator and design strategy are:

- First and foremost: get clear about the design goals, i.e. what property of an oscillator do you want to exploit and why?

- Is direct access to the phase or the frequency required? In other words, should they be presented as explicit variable and parameter?

- Is there a restriction in the number of state variables and complexity of integrating the system (e.g. for embedded computing)?

- Should it be possible to prove or predict analytically properties of the system (convergence, phase relationship)?

- How many elements should the system contain, i.e. is a network of oscillators needed (e.g. half-center, interneurons) or might a single oscillator be enough?
- Is there an advantage to use a strongly nonlinear oscillator or is a harmonic/phase oscillator enough, e.g. is a "neural" oscillator really the right one to achieve my goal? In modeling, does my model really concern the neural level so that the use of a neural oscillator is justified?

- Try to make the design space as orthogonal as possible (e.g. in the Matsuoka oscillator there is a strong influence on the shape if the oscillator gets coupled to others).

- Should the complexity and nonlinearity be placed into the oscillator or into the coupling (e.g. for higher order locking: either, phase oscillator and coupling which generates the higher order frequency components, or complex oscillator and simple couplings.)?

An often made assumption to treat limit cycle systems is the assumption that influence on phase and radius can be completely separated, this assumption directly follows out of the stability directions as discussed. However, a perturbation perpendicular to the limit cycle can in general very well have an influence on the phase and vice versa (e.g. in the van der Pol) the separation of the directions is a very useful approximation but is limited to a region "close" to the limit cycle. If we want to have a complete separation the oscillator has to be chosen accordingly, i.e. a harmonic isochronous oscillator.

Sometimes it is desired to synchronize the signal in another ratio than $1: 1$, in general oscillators can phase lock in any ratio $\mathrm{n} / \mathrm{p} n, p \in \mathbb{N}$. However, harmonic oscillators are not sufficient to achieve this task unless the input contains higher harmonics (ideally pulse like). Relaxation oscillators can phase lock with other ratios to a harmonic signal.

Finally, it is important to stress that there are many design aspects which in this article could not be discussed. As an example consider transient time for locking. It turns out that relaxation oscillators are well suited for rapid phase locking (Somers \& Kopell, 1993), this is due to the bent isochrones, i.e. even a small perturbation can drive oscillator over many isochrones and thus advance it rapidly in towards a stable phase. We see that also for such discussions the basics are the topics discussed in the paper.

We do not address another way of distinguishing two dynamical systems namely by their bifurcation behavior. In dynamical systems it is a typical phenomenon that if some parameters are changed beyond a critical value the qualitative behavior of the system can completely change, e.g. an oscillator can bifurcate to fixed point behavior. If two systems differ in their bifurcation behavior it is usually not possible to transform one into the other by only a change of coordinate systems. Different oscillators can have different bifurcation behavior. It is important to realize, that we do only discuss the oscillators in their oscillatory regime, far away from the bifurcation points and the above made statements are only valid in this parameter range. 
Furthermore, we have simplified the discussion by the fact that we do only concentrate on the limit cycle attractor of the system, while it can possibly have other attractors. Thus our discussion is limited to the basin of attraction of the limit cycle attractor (e.g. every limit cycle encloses a unstable fixed point from which the solutions would not converge to the limit cycle).

Outlook and future research It is an immense task to classify the design of oscillators and many details could not be discussed in this article.

We are convinced that taking an engineering perspective on oscillators is needed in order to make full use of them in robotics applications. This paper is only a first step in that direction.

It is possible that an engineered system might lack some of the self-organization properties and flexibility of natural oscillatory networks, but this is a fundamental problem when trying to exploit systems capable of self-organization to engineering. On the other hand we gain methodology, guarantees, but clearly such questions are open for research, see (Buchli \& Santini, 2005) for a deeper discussion.

There are many substrates other than digital computers which allow for structurally stable oscillators, i.e. chemical oscillators, (analog) electronic, biological (see also (Buchli \& Santini, 2005)). Choosing such a substrate however narrows down the degrees of freedom in the design, but still the key to understanding and engineering those systems is presented in this article. It would of course be interesting to exploit such substrates for engineering applications.

\section{Acknowledgments}

This work is funded by the Swiss National Science Foundation (A.I. \& J.B.) and by the European Commission's Cognition Unit, project no. IST-2004-004370: RobotCub (L.R.).

\section{References}

Buchli, J., Iida, F., \& Ijspeert, A. (2006). Finding resonance: Adaptive frequency oscillators for dynamic legged locomotion. In Proceedings iros 2006. (In press.)

Buchli, J., \& Ijspeert, A. (2004a). Distributed central pattern generator model for robotics application based on phase sensitivity analysis [conference]. In A. Ijspeert, M. Murata, \& N. Wakamiya (Eds.), Biologically inspired approaches to advanced information technology: First international workshop, BioADIT 2004 (Vol. 3141, pp. 333-349). Springer Verlag Berlin Heidelberg.
Buchli, J., \& Ijspeert, A. (2004b). A simple, adaptive locomotion toy-system. In S. Schaal, A. Ijspeert, A. Billard, S. Vijayakumar, J. Hallam, \& J. Meyer (Eds.), From animals to animats 8. proceedings of the eighth international conference on the Simulation of Adaptive Behavior (sab'04) (pp. 153-162). MIT Press.

Buchli, J., Righetti, L., \& Ijspeert, A. (2005). A dynamical systems approach to learning: a frequencyadaptive hopper robot [conference]. In Proceedings of the VIIIth european conference on artificial life ECAL 2005 (pp. 210-220). Springer Verlag.

Buchli, J., \& Santini, C. (2005). Complexity engineering: Harnessing emergent phenomena as opportunities for engineering [misc]. In Reports of the santa fe institute's complex systems summer school 2005. Santa Fe Institute.

Collins, J., \& Richmond, S. (1994). Hard-wired central pattern generators for quadrupedal locomotion. Biological Cybernetics, 71 (5), 375-385.

Crawford, J. (1991). Introduction to bifurcation theory. Rev Mod Phys, 63(4), 991-1037.

Delcomyn, F. (1980). Neural basis for rhythmic behaviour in animals. Science, 210, 492-498.

Endo, G., Nakanishi, J., Morimoto, J., \& Cheng, G. (2005). Experimental studies of a neural oscillator for biped locomotion with q rio. In Proceedings of the 2005 ieee international conference on robotics a nd automation (pp. 598-604). Barcelona, Spain.

Ermentrout, B. (1991). An adaptive model for synchrony in the firefly pteroptyx malaccae. J. Math. Biol., 29, 571-585.

Ermentrout, G., \& Kleinfeld, D. (2001). Traveling electrical waves in cortex: Insights from phase dynamics and speculation on a computational role. Neuron, 29, 33-44.

Ermentrout, G., \& Kopell, N. (1994). Inhibitionproduced patterning in chains of coupled nonlinear oscillators. SIAM J.Appl. Math., 54(2), 478-507.

FitzHugh, R. (1961). Impulses and physiological states in theoretical models of nerve membrane. Biophysical J., 445-466.

Fukuoka, Y., Kimura, H., \& Cohen, A. (2003). Adaptive dynamic walking of a quadruped robot on irregular terrain based on biological concepts. The International Journal of Robotics Research, 3-4, 187-202.

Galicki, M., Leistritz, L., \& Witte, H. (1999). Learning continous trajectories in recurrent neural networsk with time-dependent weights. IEEE Transactions on Neural Networks, $10(4)$.

Glass, L., \& Mackey, M. (1988). From clocks to chaos, the rythms of life. Princeton University Press.

Golubitsky, M., \& Stewart, I. (2006). Nonlinear dynamics of networks: the groupoid formalism. Bull. Amer. Math. Soc.

Grillner, S. (1985). Neurobiological bases of rhythmic motor acts in vertebrates. Science, 228 (4696), 143- 
149.

Haken, H. (1983). Synergetics. an introduction (3rd ed.). Springer Verlag Berlin Heidelberg.

Hodgkin, A., \& Huxley, A. (1952). Propagation of electrical signals along giant nerve fibres. Proceedings of the Royal Society of London. Series B, Biological Sciences, 140(899), 177-183.

Hopf, E. (1942). Abzweigung einer periodischen Lösung von einer stationären Lösung eines Differentialsystems. Ber. Math.-Phys., Sächs. Akad. d. Wissenschaften, Leipzig, 1-22.

Ijspeert, A. (2001). A connectionist central pattern generator for the aquatic and terrestrial gaits of a simulated salamander. Biological Cybernetics, 84(5), 331-348.

Ijspeert, A., Crespi, A., \& Cabelguen, J. (2005). Simulation and robotics studies of salamander locomotion. Applying neurobiological principles to the control of locomotion in robots [article]. Neuroinformatics, 3(3), 171-196.

Ijspeert, A., Nakanishi, J., \& Schaal, S. (2002). Learning attractor landscapes for learning motor primitives. In Advances in neural information processing systems 15 (nips2002).

Izhikevich, E. (2001). Resonate-and-fire neurons. Neural Networks, 14, 883-894.

Kay, B., Kelso, J., Saltzman, E., \& G., S. (1987). Spacetime behavior of single and bimanual rhythmical movements: Data and limit cycle model. ournal of Experimental Psychology: Human Perception 8 Performance, 13(2), 178-192. (a lot of dots missing, see also kay87html.pdf)

Kimura, H., Akiyama, S., \& Sakurama, K. (1999). Realization of dynamic walking and running of the quadruped using neural oscillator. Auton. Robot., 7, $247-258$.

Kopell, N. (1988). Neural control of rhythmic movements in vertebrates. John Wiley and Sons, New York.

Kopell, N., \& Ermentrout, G. (1988). Coupled oscillators and the design of central pattern generators. Mathematical Biosciences, 90(1-2), 87-109.

Kramer, M., Herschel, R., \& Calo, J. (1984). Sensitivity analysis of oscillatory systems. Applied Mathematical Modelling, 8(5), 328-340.

Kuramoto, Y. (1984). Chemical oscillations, waves, and turbulence. Springer Verlag Berlin Heidelberg.

Large, E. (1994). Resonance and the perception of musical meter. Connection Science, 6(2-3), 177-208.

Large, E. (1996). Modeling beat perception with a nonlinear oscillator. In Proceedings of the eighteenth annual conference of the cognitive science society.

Leistritz, L., Galicki, M., Witte, H., \& Kochs, E. (2002). Training trajectories by continuous recurrent multilayer networks. IEEE Transactions on Neural Networks, 13(2), 283-291.

Marbach, D., \& Ijspeert, A. (2005). Online optimization of modular robot locomotion [conference]. In Pro- ceedings of the ieee int. conference on mechatronics and automation (icma 2005) (pp. 248-253).

Matsuoka, K. (1985). Sustained oscillations generated by mutually inhibiting neurons with adaptation. Biol. Cybern., 52, 367-376.

Matsuoka, K. (1987). Mechanisms of frequency and pattern control in the neural rhytm generators. Biol. Cybern., 56, 345-353.

Morimoto, J., Endo, G., Nakanishi, J., Hyon, S., Cheng, G., Bentivegna, D., \& Atkeson, C. (2006). Modulation of simple sinusoidal patterns by a coupled oscillator model for biped walking. In Proceedings of the 2006 ieee international conference on robotics and automation (pp. 1579-1584).

Nishii, J. (1998). A learning model for oscillatory networks. Neural Networks, 11(2), 249-257.

Nishii, J. (1999). Learning model for coupled neural oscillators. 10, 10, 213-226.

Okada, M., Nakamura, D., \& Nakamura, Y. (2003). Hierachical design of dynamics based information processing system for humanoid motion generation. In Proceedings amam 2003.

Okada, M., Tatani, K., \& Nakamura, Y. (2002). Polynomial design of the nonlinear dynamics for the brain-like information processing of whole body motion. In Proceedings of icra 2002 (Vol. 2, pp. 1410-1415).

Pikovsky, A., Rosenblum, M., \& Kurths, J. (2001). Synchronization, a universal concept in nonlinear sciences (Vol. 12). Cambridge University Press, Cambridge, UK.

Righetti, L., Buchli, J., \& Ijspeert, A. (2006). Dynamic hebbian learning in adaptive frequency oscillators [article]. Physica D, 216(2), 269-281.

Righetti, L., \& Ijspeert, A. (2006a). Design methodologies for central pattern generators: application to crawling humanoids. In Proceedings rss 06.

Righetti, L., \& Ijspeert, A. (2006b). Programmable central pattern generators: an application to biped locomotion control [conference]. In Proceedings of the 2006 ieee international conference on robotics and automation.

Ruiz, A., Owens, D., \& Townley, S. (1998). Existence, learning, and replication of periodic motions in recurrent neural networks. IEEE Transactions on Neural Networks, 9(4), 651-661.

Santos, C. (2003). Attractor dynamics based generation of timed robotic trajectories. Unpublished doctoral dissertation, Universidade do Minho Escola de Engenharia, Dept. de Electronica Industrial, Guimaraes, Portugal.

Santos, C. (2004). Generating timed trajectories for an autonomous vehicle: A non-linear dynamical systems approach. In 2004 ieee international conference on robotics and automation. IEEE.

Schöner, G., Jiang, W., \& Kelso, J. (1990). A synergetic theory of quadrupedal gaits and gait transitions. 
Journal of theoretical Biology, 142, 359-391.

Schöner, G., \& Kelso, J. (1988). A synergetic theory of environmentally-specified and learned patterns of movement coordination. II Component oscillator dynamics. Biological Cybernetics, 58, 81-89.

Schöner, G., \& Santos, C. (2001). Control of movement time and sequential action through attractor dynamics: A simulation study demonstrating object interception and coordination. In Sirs 2001.

Somers, D., \& Kopell, N. (1993). Rapid synchronization through fast threshold modulation. Biological Cybernetics, 68, 393-407.

Strogatz, S. (1994). Nonlinear dynamics and chaos. with applications to physics, biology, chemistry, and engineering. Addison Wesley Publishing Company.

Strogatz, S. (2000). From kuramoto to crawford: exploring the onset of synchronization in populations of coupled oscillators. Physica D: Nonlinear Phenomena, 143(1-4), 1-20.

Taga, G. (1994). Emergence of bipedal locomotion through entrainment among the neuro-musculoskeletal system and the environment. Physica D: Nonlinear Phenomena, 75(1-3), 190-208.

Taga, G. (1995a). A model of the neuro-musculo-skeletal system for human locomotion. ii real-time adaptability under various constraints. Biological Cybernetics, 73(2), 113-121.

Taga, G. (1995b). A model of the neuro-musculo-skeletal system for human locomotion. I. Emergence of basic gait. Biological Cybernetics, 73(2), 97-111.

Taga, G. (1998). A model of the neuro-musculo-skeletal system for anticipatory adjustment of human locomotion during obstacle avoidance. Biological $\mathrm{Cy}$ bernetics, 78(1), 9-17.

Wang, W., \& Slotine, J. (2005). On partial contraction analysis for coupled nonlinear oscillators. Biological Cybernetics, 92(1), 38-53.

Wilbur, C., Vorus, W., Cao, Y., \& Currie, S. (2002). Neurotechnology for biomimetic robots. Bradford/MIT Press, Cambridge London.

Williamson, M. (1998). Exploiting natural dynamics in robot control. In Proceedings fourth european meeting on cybernetics and systems research (emcsr' '98).

Winfree, A. (2001). The geometry of biological time (2nd ed.). Springer Verlag.

Zegers, P., \& Sundareshan, M. (2003). Trajectory generation and modulation using dynamic neural networks. IEEE Transactions on Neural Networks, $14(3), 520-533$. 


\begin{tabular}{|c|c|c|c|c|c|c|}
\hline \multirow[b]{2}{*}{ contribution } & \multirow[b]{2}{*}{$\mathrm{R} / \mathrm{A}$} & \multicolumn{3}{|c|}{ offline } & \multicolumn{2}{|c|}{ online } \\
\hline & & $\begin{array}{l}\text { "by } \\
\text { hand" }\end{array}$ & $\begin{array}{l}\text { algo- } \\
\text { rithmic }\end{array}$ & dynamic & $\begin{array}{l}\text { algo- } \\
\text { rithmic }\end{array}$ & dynamic \\
\hline $\begin{array}{l}\text { (Ermentrout \& Kopell, 1994) } \\
\text { (Williamson, 1998) } \\
\text { (Ijspeert et al., 2005) } \\
\text { (Matsuoka, 1985, 1987) } \\
\text { (Schöner, Jiang, \& Kelso, 1990) } \\
\text { (Schöner \& Kelso, 1988) } \\
\text { (Endo et al., 2005) } \\
\text { (Morimoto et al., 2006) } \\
\text { (Righetti \& Ijspeert, 2006a) } \\
\text { (Taga, 1994, 1995b, 1995a) } \\
\text { (Buchli \& Ijspeert, 2004a) } \\
\text { (22, 2003) } \\
\text { (Schöner \& Santos, 2001; Santos, 2003, 2004) } \\
\text { (Collins \& Richmond, 1994) } \\
\text { (Ijspeert, 2001) } \\
\text { (Zegers \& Sundareshan, 2003) } \\
\text { (Okada, Tatani, \& Nakamura, 2002; Okada, Nakamura, \& } \\
\text { Nakamura, 2003) } \\
\text { (Ijspeert, Nakanishi, \& Schaal, 2002) } \\
\text { (Ruiz, Owens, \& Townley, 1998) } \\
\text { (Galicki, Leistritz, \& Witte, 1999; Leistritz, Galicki, Witte, \& } \\
\text { Kochs, 2002) } \\
\text { (Righetti \& Ijspeert, 2006b) } \\
\text { (Marbach \& Ijspeert, 2005) } \\
\text { (Nishii, 1999) } \\
\text { (Ermentrout, 1991) } \\
\text { (Large, 1994, 1996) } \\
\text { (Buchli \& Ijspeert, 2004b; Righetti, Buchli, \& Ijspeert, 2006; } \\
\text { Buchli, Righetti, \& Ijspeert, 2005; Buchli et al., 2006) }\end{array}$ & $\begin{array}{l}\mathrm{R} \\
\mathrm{R} \\
\mathrm{R} \\
\mathrm{R} \\
\mathrm{R} \\
\mathrm{R} \\
\mathrm{R} \\
\mathrm{R} \\
\mathrm{R} \\
\mathrm{R} \\
\mathrm{R} \\
\mathrm{R} \\
\mathrm{R} \\
\mathrm{R} \\
\mathrm{R} \\
\mathrm{R} \\
\mathrm{R} \\
\\
\mathrm{R} \\
\mathrm{R} \\
\mathrm{R} \\
\mathrm{R} \\
\mathrm{A} \\
\mathrm{A} \\
\mathrm{A} \\
\mathrm{A} \\
\mathrm{A}\end{array}$ & $\begin{array}{l}1 \mathrm{a} \\
1 \mathrm{a} \\
1 \mathrm{a} \\
1 \mathrm{a} \\
1 \mathrm{a} \\
1 \mathrm{a} \\
1 \mathrm{a} \\
1 \mathrm{a} \\
1 \mathrm{~b} \\
1 \mathrm{a} \\
1 \mathrm{a} \\
1 \mathrm{a} \\
1 \mathrm{a} \\
1 \mathrm{a}\end{array}$ & $\begin{array}{l}1 \mathrm{a} / 2 \\
2 \mathrm{~b} \\
2 \mathrm{a} \\
2 \mathrm{~b} \\
2 \\
2\end{array}$ & $1 \mathrm{a} / 2 \mathrm{~b}$ & $1 \mathrm{a}$ & $\begin{array}{l}1 \mathrm{c} / 1 \mathrm{a} \\
1 \mathrm{c} \\
1 \mathrm{c} \\
1 \mathrm{c}\end{array}$ \\
\hline
\end{tabular}

Table 3 Table classifying some of the contribution of the field oscillators applied to robotics and related modeling. The contributions are classified according the the design method as discussed in Section 4. The labels in each case correspond the design goals identified in Section 3: (1) Timing: Design that influence the phase of the oscillator: (a) influence on (relative) phase, (b) instantaneous frequency, and (c) average frequency. (2) Design that influences the geometry of the oscillator: (a) influences on $r,(b)$ the design of $\mathbb{T}^{-1} /$ output filter. The column " $\mathrm{R} / \mathrm{A}$ " indicates whether the resulting system is reactive or adaptive. This table has sparsely populated columns which point to open research question. 